\title{
ZS Research Square

\section{Risk Factors for Dysphagia After Anterior Cervical Discectomy and Fusion With the Zero-P Implant System: a Retrospective Study With Minimum of 2 Years Follow-up}

\author{
Rong Xue \\ Xinghua Hospital \\ Ningdao Li \\ Shenzhen Luohu Hospital \\ Zhurong Ji \\ Xinghua Hospital \\ Xingdong Cheng \\ Xinghua Hospital \\ Zhuqiu Zhang \\ Xinghua Hospital \\ Feng Zhang ( $\square$ zhangfeng@126.com ) \\ Xinghua Hospital
}

Research article

Keywords: risk factors, dysphagia, ACDF, cage and plate, Zero-P

Posted Date: January 5th, 2021

DOl: https://doi.org/10.21203/rs.3.rs-138933/v1

License: (a) (i) This work is licensed under a Creative Commons Attribution 4.0 International License.

Read Full License

Version of Record: A version of this preprint was published at Orthopaedic Surgery on November 28th, 2021. See the published version at https://doi.org/10.1111/os.13170. 


\section{Abstract}

Background: Dysphagia is one of the most common complications after anterior cervical spine surgery. The study aimed to evaluate the risk factors for dysphagia after anterior cervical discectomy and fusion (ACDF) with the Zero-P Implant System by multidimensional analysis and investigated the predictive values of these risk factors for dysphagia.

Methods: A retrospective analysis of 260 patients who underwent ACDF with the Zero-P Implant System and had at least 2 year of follow-up ware performed. All patients were divided into a non-dysphagia group and a dysphagia group. Sex, age, body mass index (BMI), intraoperative time, estimated blood loss, diabetes mellitus, hypertension, smoking, alcohol consumption, prevertebral soft-tissue thickness, the levels of surgery, $\mathrm{O}-\mathrm{C} 2$ angle, $\mathrm{C} 2-7$ angle, $\mathrm{T} 1$ slope and segmental angle were analyzed. Chi-square test and logistic regression were performed to analyze the predictive value of each dimension for dysphagia.

Results: In total, the non-dysphagia group comprised 170 patients and the dysphagia group comprised 90 patients. Chi-square test results indicated that number of operated levels, operation time dT1 slope, dO-C2 angle, dC2-7 angle, segmental angle and dPSTT were associated with a high incidence of dysphagia. Multivariate logistic regression analysis showed that number of operated levels, operation time, $\mathrm{dC} 2-7$ angle and dPSTT were significantly associated with postoperative dysphagia.

Conclusions: Number of operated levels, operation time, dC2-7 angle and dPSTT were significantly associated with postoperative dysphagia. In additionally, sufficient preoperative preparation, evaluation combining with proficient and precise treatment measures are suggested to reduce the incidence of postoperative dysphagia when ACDF is performed.

\section{Introduction}

Cervical spondylotic myelopathy and cervical spondylotic radiculopathy are the most common spinal disorders. Surgical treatment is indicated when conservative therapy fails or when the symptoms worsen. Anterior cervical discectomy and fusion (ACDF) is the gold standard for the treatment of cervical spondylosis. ${ }^{1-4}$ Anterior cage-plate construct is commonly used in ACDF in order to enhance segmental stability improve cervical sagittal alignment, reduce graft extrusion and subsidence, and increase fusion rates. These techniques have their own benefits as well as potential drawbacks and adverse effects. The most often mentioned shortcomings of these techniques are the breakage or loosening of plate and screws, trachea-esophageal injury, neurovascular injury, and postoperative dysphagia have caused concerns. $^{5}$

Dysphagia has been considered one of the most common complications after anterior cervical spine surgery. ${ }^{6-9}$ Many studies have reported that an anterior plate with a lower, smoother profile may reduce the incidence of dysphagia after ACDF. To reduce these complications, the Zero-P has been introduced and applied for ACDF. The device can be implanted into the intervertebral space entirely, providing adequate stability and avoiding implant contact with the prevertebral soft tissue. 
Although the risk factors for postoperative dysphagia are multidimensional, the literature lacks a comprehensive analysis of them. This study assessed the incidence of dysphagia after anterior cervical spine surgery with the Zero-P implant system and investigated the predictive values of the risk factors for dysphagia.

\section{Materials And Methods}

A retrospective analysis was performed in our hospital from January 2014 to July 2017. This retrospective study was approved by the Ethics Committee of our Hospital. All of the patients were recruited after providing informed consent for analysis of their clinical data. Radiologic diagnoses were established in each patient through routine preoperative cervical anteroposterior, lateral, flexion-extension radiographs and cervical magnetic resonance imaging or computed tomography scans. All patients had symptoms and signs of neural compression that were refractory to conservative treatment. All patients were undergone ACDF with Zero-P. Patients were divided into the postoperative dysphagia group and the non-dysphagia group. Sex, age, BMI, diabetes, hypertension, smoking, alcohol consumption, duration of symptom, surgery blood loss, degree of prevertebral soft tissue swelling, and 0-C2 angle, C2-7 angle, T1 slope, the operative segment and the number of operative segments were collected.

\section{Inclusion and Exclusion Criteria of patients}

Inclusion criteria: (1) Patients were diagnosed as cervical spondylotic myelopathy or cervical spondylotic radiculopathy; (2) The clinical symptoms and signs of the disease were consistent with the results of imaging examination; (3) Conservative treatment was not effective for more than 6 months; (4) ACDF is suitable for the segment, the fused segment prosthesis was Zero-P; (5) Preoperative swallowing was normal; (6) The follow-up time was at least 2 year.

Exclusion criteria: (1) Preoperative dysphagia; (2) A history of other disorders that may trigger symptoms of dysphagia, such as craniocerebral trauma or cerebral infarction; (3) Received revision surgery; (4) Combined with cervical deformity, tumor, severe osteoporosis, ankylosing spondylitis and rheumatoid arthritis; (5) Incomplete imaging data; (6) loss of follow-up or follow-up was less than 1 year; (7) Received hybrid surgery.

\section{Surgical procedure}

All the surgical procedures were performed by the same senior surgeon using standard Smith-Robinson approach. The cartilaginous disc endplate was carefully removed and care was taken to avoid excessive damage to bony endplate. The posterior osteophytes were removed by curettes and Kerrison rongeurs. After complete decompression of the spine cord and nerve roots, the ideal sizes of cages were selected by radiographic assisted trials. The width of the cage was determined by the distance between the two Luschka's joints, and the height of the cage was determined by different trails under radiography, when trails are tightly fitted in the disc space without over-distraction of the disc space or facet joints. Porous bioceramic artificial bone was used to fill in the cage in all patients. Proper sized devices (Zero-P, USA) or 
cages along with anterior cervical plate were inserted and the anchorage systems were inserted in the vertebral bodies under the fluoroscopic guidance.

Postoperatively, all patients received prophylactic anti-infective therapy for 2 days. Methylprednisolone was administered intravenously for 3 days, with doses being $40 \mathrm{mg}, 20 \mathrm{mg}$, and $10 \mathrm{mg}$. Drainage fluid amount and characteristics were monitored. The drainage tube was removed within 24 to 48 hours postoperatively. All patients were treated with cervical collar fixation for 3 months.

\section{Evaluation Criteria}

All patients were followed up for at least 24 months after reoperation. Data were collected preoperatively and at $3,6,12,24$ months and final follow-up after surgery.

Clinical evaluation was including: (1) The modified Japanese Orthopaedic Association (JOA) scoring system was used to determine functional status before surgery and the final follow-up visit. (2) The recovery rate (\%) at the final follow-up visit was calculated by using the Hirabayashi method: (postoperative JOA score - preoperative score) / (17 - preoperative score) $\times 100 \%$. And the recovery rates were graded as follows: $75 \%$ and greater, excellent; $50 \%$ to $74 \%$, good; $25 \%$ to $49 \%$, fair; and less than $25 \%$, poor. (3) Neck Disability Index (NDI) was used to evaluate how much the neck pain affected the ability to manage daily life.

Radiographic assessment was including: (1) C2-7 lordosis: the angle between the C2 and C7 lower endplates; (2) segmental lordosis: the angle between the upper endplate of the uppermost body and the lower endplate of the lowermost body at the operated segments; (3) T1 slope: the angle between the T1 upper endplate and the horizontal reference line; (4) O-C2 angle (O-C2A) was the angle between the McGregor's line and the inferior margin of the $\mathrm{C} 2$ vertebrae; (5) prevertebral soft-tissue thickness (PSTT). ${ }^{10,11}$ Furthermore, the differences of parameters before and after operation were analyzed. (dC27 angle, dSL, dO-C2 angle, dT1 slope and dPSTT)

The Bazaz grading system was chosen to evaluate dysphagia after surgery. The scores of the Bazaz grading system were ranked as follows: 0-none, 1-mild, 2-moderate and 3-severe, representing no episodes of swallowing problems, rare episodes of dysphagia, occasional swallowing difficulties with specific foods and frequent swallowing difficulties with most foods, respectively. ${ }^{12}$

\section{Statistical analysis}

All statistical analyses were performed with the statistical program SPSS version 22.0 (SPSS Inc., Chicago, IL, USA). Continuous variables were expressed as mean \pm standard deviation. A paired t-test was used to analyze changes between pre- and postoperative parameters. An independent t-test was used to compare quantitative variables between the two groups. A chi-square test was used to compare qualitative data between the two groups. To eliminate the influence of confounding factors, ordinal logistic regression was performed for multifactor regression of factors with a $P$ value less than 0.5 in the 
single factor analysis. The results were regarded as significant when the P-values were less than 0.05 in this study.

\section{Results}

In total, 260 consecutive patients were enrolled in this study, including 109 males and 151 females with an average age of $58.33 \pm 4.68$ years (ranging, $42-82$ years). These patients were followed up for $18.5 \pm$ 3.5 months (range, 14-32 months). The diagnoses were cervical spondylotic myelopathy in 105 cases, cervical spondylotic radiculopathy in 75 cases, mixed cervical spondylosis in 34 cases, and posterior longitudinal ligament ossification in 46 cases.

A total of 70 patients developed dysphagia after surgery. The main symptoms included difficulty in swallowing or an inability to swallow when swallowing liquids; food retention in the throat fatigue during swallowing or an inability to swallow solids and pain or a burning sensation with swallowing. According to the Bazaz dysphagia grading system, mild, moderate, and severe dysphagia were found in 50,17 , and 3 patients, respectively. In total, 37.1\% $(n=26)$ had resolved by 3 month, 38.6\% $(n=27)$ by 6 months, and $17.1 \%(n=12)$ by 12 months. 5 patients underwent esophageal angiography, with no significant esophageal stenosis or esophageal fistula observed, and 4 patients underwent electronic fiber laryngoscopy, which showed no significant laryngeal mucosal injury, vocal cord relaxation, or paralysis. At final review, dysphagia was still present in 4 patients (5.8\%), including 3 patients with mild dysphagia and 1 patient with moderate dysphagia. (Table 1)

Table 1

Summary of Dysphagia and Dysphonia Data

\begin{tabular}{|llll|}
\hline Postoperative Time & \multicolumn{3}{l|}{ Dysphagia } \\
\cline { 2 - 4 } & Mild & Moderate & Severe \\
\hline Postoperative & 50 & 17 & 3 \\
\hline 3 months & 31 & 10 & 3 \\
6 months & 12 & 4 & 1 \\
\hline 12 months & 3 & 2 & 0 \\
\hline 24 months & 2 & 2 & 0 \\
\hline
\end{tabular}

The dysphagia group consisted of 70 consecutive patients while the non-dysphagia group consisted of 190 consecutive patients. For clinical outcomes, both groups demonstrated significant improvement in the NDI and JOA scores $(P<0.001)$. We used univariate analysis to evaluate the independent factors, including sex, age, duration of symptom, BMI, operation time, blood loss, smoke, operated levels, alcohol abuse and BMI to see which were related to postoperative dysphagia. Chi-square test results indicated that number of operated levels and operation time were associated with a high incidence of dysphagia. 
(Table 2). Furthermore, imaging parameters including dO-C2 angle, dC2-7 angle, dT1 slope, segmental angle and dPSTT were also evaluated. The result indicated that dT1 slope, dO-C2 angle, dC2 -7 angle, segmental angle and dPSTT were related to postoperative dysphagia. (Table 3)

Table 2

Demographic and Clinical characteristics

\begin{tabular}{|lll|}
\hline Item & Dysphagia group & None-Dysphagia group \\
\hline Patients & 70 & 190 \\
\hline Age (y) & $59.2 \pm 8.2$ & $57.7 \pm 5.9$ \\
\hline Gender: male & 34 & 75 \\
\hline Duration of symptoms (m) & $20.9 \pm 3.6$ & $22.1 \pm 3.2$ \\
\hline Smoke & 12 & 29 \\
\hline Alcohol Abuse & 11 & 32 \\
\hline Follow-up (m) & $19.7 \pm 3.7$ & $17.1 \pm 5.5$ \\
\hline Number of operated levels & $3.2 \pm 0.7$ & $1.4 \pm 0.5^{\star}$ \\
\hline Operation time (min) & $109.4 \pm 20.3$ & $73.2 \pm 15.6^{\star}$ \\
\hline Blood loss (ml) & $60.1 \pm 22.2$ & $40.9 \pm 18.3$ \\
\hline Pre-OP NDI & $2.02 \pm 1.41$ & $2.19 \pm 1.54$ \\
\hline Prost-OP NDI & $1.89 \pm 2.16$ & $2.01 \pm 1.73$ \\
\hline Pre-OP JOA & $10.5 \pm 1.1$ & $10.7 \pm 1.5$ \\
\hline Post-OP JOA & $15.7 \pm 1.5$ & $15.8 \pm 1.2$ \\
\hline Recover Ratio of JOA (\%) & $72.6 \pm 16.1$ & $71.1 \pm 23.1$ \\
\hline Outcome (excellent/good, \%) & 100 & 100 \\
\hline *: There was a statistically significant difference between two group, P value $<0.05$ \\
\hline
\end{tabular}


Table 3

Imaging Parameters between the 2 groups.

\begin{tabular}{|lll|}
\hline Item & Dysphagia group & None-Dysphagia group \\
\hline Pre-OP O-C2 angle $\left(^{\circ}\right)$ & $16.2 \pm 6.4$ & $16.5 \pm 4.5$ \\
\hline Prost-OP O-C2 angle $\left(^{\circ}\right)$ & $14.2 \pm 3.1$ & $14.3 \pm 3.2$ \\
\hline Pre-OP C2-7 angle $\left(^{\circ}\right)$ & $15.1 \pm 8.6$ & $15.4 \pm 7.9$ \\
\hline Post-OP C2-7 angle $\left(^{\circ}\right)$ & $16.3 \pm 6.1$ & $16.1 \pm 6.2$ \\
\hline Pre-OP T1 slope $\left(^{\circ}\right)$ & $26.2 \pm 4.7$ & $26.4 \pm 4.7$ \\
\hline Post-OP T1 slope $\left(^{\circ}\right)$ & $30.4 \pm 6.7$ & $26.4 \pm 6.7 *$ \\
\hline Pre-OP Segmental angle $\left(^{\circ}\right)$ & $7.5 \pm 6.9$ & $7.5 \pm 6.3$ \\
\hline Post-OP Segmental angle $\left(^{\circ}\right)$ & $14.5 \pm 6.2$ & $8.2 \pm 5.9 *$ \\
\hline Pre-OP PSTT $(\mathrm{mm})$ & $9.2 \pm 1.5$ & $9.3 \pm 1.4$ \\
\hline Pre-OP PSTT $(\mathrm{mm})$ & $13.6 \pm 3.7$ & $10.9 \pm 1.0 *$ \\
\hline *: There was a statistically significant difference between two group, P value $<0.05$ \\
\hline
\end{tabular}

Multivariate logistic regression analysis showed that number of operated levels, operation time, $\mathrm{dC} 2-7$ angle and dPSTT were significantly associated with postoperative dysphagia. However, dT1 slope, dO-C2 angle and dSL was not significantly associated with postoperative dysphagia in the multivariate model (Table 4).

Table 4

The results of the logistic regression analysis between related factors and dysphagia

\begin{tabular}{|llll|}
\hline Clinical Factors & $\mathbf{P}$ & Odds Ratio & $\mathbf{9 5 \%} \mathbf{C l}$ \\
\hline number of operated levels & 0.037 & 1.917 & $1.0-3.3$ \\
\hline operation time & $<0.001$ & 2.46 & $2.0-5.6$ \\
\hline dO-C2 angle & 0.022 & 0.123 & $1.9-7.3$ \\
\hline dC2-7 angle & 0.041 & 2.323 & $0.6-3.1$ \\
\hline dT1 slope & 0.215 & 2.113 & $1.9-4.9$ \\
\hline segmental angle & 0.169 & 3.211 & $1.0-3.3$ \\
\hline dPSTT & $<0.001$ & 2.063 & $3.3-8.3$ \\
\hline
\end{tabular}

\section{Discussion}


Dysphagia contributes to higher self-reported disability and lower physical health status. The most probable explanation for postoperative dysphagia is that it is a multifactorial phenomenon, explained by esophageal retraction, direct cervical plate stimulating the esophagus, prevertebral swelling, among others. Persistent and severe dysphagia may lead to various degrees of discomfort, and increase the risk of various complications such as such as difficulty in eating or drinking, bronchospasm, aspiration pneumonia, dehydration, asphyxia and malnutrition. However, the pathophysiology and risk factors of postoperative dysphagia are not fully understood.

The incidence of dysphagia after anterior cervical spine surgery has been reported as ranging from 1$80 \%$. Baron et al believed that the incidence of transient dysphagia after anterior cervical fusion was as high as $80 \%$ and that the symptoms of most patients were relieved after treatment. ${ }^{13}$ These greatly varying incidence rates may be related to factors such as Surgical approach, sample size, and differences in case selection and evaluation methods, particularly regarding the criteria used to define postoperative dysphagia. ${ }^{14,15}$ Theoretically, if an anterior cervical titanium plate is placed directly into the esophagus, the titanium plate may affect the incidence of postoperative dysphagia. Any mechanical irritation or impingement of the esophagus may cause symptoms of dysphagia. No-notch implants were considered to be associated with anterior plate + cage. Our result showed that the incidence of dysphagias of patients used Zero-P system was lower than that of the previous reported.

Many scholars have developed classification systems to define and classify postoperative dysphagia, but the inconsistent usage and lack of consensus limit its research progress. X-ray fluoroscopic examination of swallowing function has long been regarded as the gold standard for assessing swallowing difficulty. The Bazaz grading system is widely used to assess the incidence of dysphagia after cervical spine surgery. But it has many shortcomings. This scale is based on qualitative information collected by an investigator to assess the patient's subjective sensation of difficulty when swallowing liquids and solids, possible sensory disruptions causing postoperative dysphagia may be challenging to explain and may not reflect accurate clinical outcomes. In addition, the correlation between the indicators of dysphania and subjective performance is not clear. Anderson believed that the self-assessment questionnaire for patients' main symptoms might be a relatively reliable method for evaluating clinical dysphagia after anterior cervical surgery. ${ }^{16}$ SWAL QOL score was recommended to be used to assess the degree of dysphagia. Postoperative dysphagia was evaluated using the Bazaz scoring system in our result, and it was found that dysphagia was a common early complication after anterior cervical surgery, and the incidence and severity of dysphagia gradually decreased over time. After 1 year of follow-up, almost all patients had resolved their dysphagia, and only a few still had mild dysphagia. Bazaz's prospective study of 249 patients who underwent anterior cervical surgery found that the incidence of dysphagia was $50.2 \%, 32.2 \%, 17.8 \%$, and $12.5 \%$ at $1,2,6$, and 12 months after surgery, respectively. At 6 months after surgery, only $4.8 \%$ of patients had moderate or severe dysphagia, which was basically consistent with the results of this study.

Recently, several studies have compared the clinical outcomes spacer and cervical sagittal balance in ACDF for treating cervical disorders. ${ }^{17-19}$ And the result of some studies indicated the Cobb angle, T1 
slope and some other parameters are closely related to clinical outcomes. ${ }^{20-22}$ To the best of our knowledge, few reports have described the effect of the difference between postoperative and preoperative sagittal balance on postoperative dysphagia after ACDF with Zero-P. Consequently, one objective of this retrospective analysis was to summarize and identify the effect of the cervical sagittal balance and other possible related factors on dysphagia. And provides for future spinal surgeries with evidence on how to reduce the incidence of dysphagia after ACDF with Zero-P.

Miyata believed that posterior cervical surgery, such as posterior occipito-cervical fusion, if the surgery changes the physiological curvature, it will result in mechanical strictures of oropharynx, which may lead to postoperative swallowing disorders. ${ }^{23}$ This provides an idea for us to study the swallowing disorder caused by the overall curvature change after cervical spine surgery. Our study indicated that dO-C2 angle and $\mathrm{dC} 2-7$ angle were significantly related with postoperative dysphagia.

Khaki believed that anterior cervical surgery could affect the throat stage in the four stages of swallowing process, thus leading to postoperative dysphagia. ${ }^{24}$ Based on the previous studies, we concluded that the posterior pharyngeal wall protruded forward due to the large angle of dC2-7 angle after surgery, which reduced the throat volume and affected the squeezing effect of pharynx during eating, thus leading to dysphagia. However, there is still a lack of direct imaging evidence to confirm this view, and the hypothesis proposed by us can only explain to a certain extent the mechanism of the dC2-7 angle and postoperative dysphagia. In the anterior cervical decompression, the intervertebral space should be extended as far as possible with the use of a retractor to restore the normal curvature of the cervical spine and reduce postoperative cervical degeneration. The results of this study suggest that excessive expansion of cervical spine space may cause excessive changes in C2-7 Angle, which may lead to forward protrusion of the posterior pharyngeal wall, resulting in postoperative dysphagia. Therefore, the recovery of anatomical force line should not be pursued only, but also the change of cervical curvature should be controlled to reduce the occurrence of postoperative dysphagia in ACDF. This suggests that intraoperative control of $\mathrm{C} 2-\mathrm{C} 7$ angle within a reasonable range may reduce the probability of postoperative dysphagia symptoms.

Operative time was another factor associated with dysphagia in our study. In a prospective study of 38 patients undergoing single-segment and double-segment ACDF surgery, found that extended duration of surgery was the only variable associated with the severity of dysphagia 12 weeks after surgery. ${ }^{25} \mathrm{We}$ hypothesized that prolonged traction of the trachea and esophagus would inevitably lead to more severe soft tissue swelling. Therefore, in complex cervical spine surgery, where the operation is expected to take a long time, surgery by a senior cervical surgeon may reduce the incidence of postoperative dysphagia more than surgery by a junior physician or resident.

There are some limitations in the current study. First, the sample size of the retrospective study was small though it was adequate to evaluate the variables. A further prospective study should be designed using a standardized scoring system and postoperative examination. Second, the follow-up period was relatively short, with a mean of 18.5 months. Therefore, the long-term clinical effect should be evaluated. Third, not 
all potential risk factors, such as the thickness and length of the plate, were not considered in the statistical analysis. In addition, the mechanisms by which the T1 slope affects the development of dysphagia after ACDF with the Zero-P implant System are not completely clear. Therefore, multicenter and randomized controlled studies are needed to verify our conclusions in the future.

\section{Conclusion}

Dysphagia is one of the most common complications after anterior cervical spine surgery. Multivariate logistic regression analysis showed that number of operated levels, operation time, dC2-7 angle and dPSTT were significantly associated with postoperative dysphagia. In additionally, sufficient preoperative preparation, evaluation combining with proficient and precise treatment measures are suggested to reduce the incidence of postoperative dysphagia when ACDF is performed.

\section{Declarations}

\section{Ethics approval}

This retrospective study was approved by the Ethics Committee of our Hospital. All of the patients were recruited after providing informed consent for analysis of their clinical data.

\section{Consent for publication}

Not applicable

\section{Availability of data and materials}

The datasets generated during and/or analyzed during the current study are publicly available. The datasets generated during and/or analyzed during the current study are available from the corresponding author on reasonable request

\section{Competing interests}

The authors declare that they have no competing interests

\section{Funding}

None

\section{Authors' contributions}

XR and LND conducted the trials and drafting the manuscript, are co-first authors. JZY and CYQ participated in the design of the study and performed the trial. ZZQ supervised and coordinated the study. All authors read and approved the final manuscript. ZF is corresponding author of this manuscript. 


\section{References}

1. Fehlings MG, Tetreault LA, Wilson JR, Skelly AC. Cervical spondylotic myelopathy: current state of the art and future directions. Spine (Phila Pa 1976). 2013;38(22 Suppl 1):S1-8.

2. Divi SN, Goyal DKC, Mangan JJ, et al. Are Outcomes of Anterior Cervical Discectomy and Fusion Influenced by Presurgical Depression Symptoms on the Mental Component Score of the Short Form12 Survey? Spine (Phila Pa 1976). 2020;45(3):201-207.

3. Khan A, Meyers JE, Blasio P, et al. Evaluation of Benefit and Cost Utility of Immediate Postanesthesia Care Unit Radiographs to Predict Airway Compromise after Anterior Cervical Discectomy and Fusion. Spine (Phila Pa 1976). 2020;Publish Ahead of Print.

4. McClure JJ, Desai BD, Shabo LM, et al. A single-center retrospective analysis of 3- or 4-level anterior cervical discectomy and fusion: surgical outcomes in 66 patients. J Neurosurg Spine. 2020:1-7.

5. Yin M, Ma J, Huang Q, et al. The new Zero-P implant can effectively reduce the risk of postoperative dysphagia and complications compared with the traditional anterior cage and plate: a systematic review and meta-analysis. BMC Musculoskelet Disord. 2016;17(1):430.

6. Riley LH, 3rd, Skolasky RL, Albert TJ, Vaccaro AR, Heller JG. Dysphagia after anterior cervical decompression and fusion: prevalence and risk factors from a longitudinal cohort study. Spine (Phila Pa 1976). 2005;30(22):2564-2569.

7. Kawamura I, Tominaga H, Tanabe F, Yamamoto T, Taniguchi N. Cervical Alignment of Anterior Cervical Hyperostosis Causing Dysphagia. Spine (Phila Pa 1976). 2019;44(5):E269-E272.

8. Vaishnav AS, Saville P, McAnany S, et al. Predictive Factors of Postoperative Dysphagia in SingleLevel Anterior Cervical Discectomy and Fusion. Spine (Phila Pa 1976). 2019;44(7):E400-E407.

9. Yew AY, Nguyen MT, Hsu WK, Patel AA. Quantitative Risk Factor Analysis of Postoperative Dysphagia After Anterior Cervical Discectomy and Fusion (ACDF) Using the Eating Assessment Tool-10 (EAT10). Spine (Phila Pa 1976). 2019;44(2):E82-E88.

10. Ota $\mathrm{M}, \mathrm{Neo} \mathrm{M}$, Aoyama T, et al. Impact of the $\mathrm{O}-\mathrm{C} 2$ angle on the oropharyngeal space in normal patients. Spine (Phila Pa 1976). 2011;36(11):E720-726.

11. Ohara A, Miyamoto K, Naganawa T, Matsumoto K, Shimizu K. Reliabilities of and correlations among five standard methods of assessing the sagittal alignment of the cervical spine. Spine (Phila Pa 1976). 2006;31(22):2585-2591; discussion 2592.

12. Bazaz R, Lee MJ, Yoo JU. Incidence of dysphagia after anterior cervical spine surgery: a prospective study. Spine (Phila Pa 1976). 2002;27(22):2453-2458.

13. Baron EM, Soliman AM, Gaughan JP, Simpson L, Young WF. Dysphagia, hoarseness, and unilateral true vocal fold motion impairment following anterior cervical diskectomy and fusion. Ann Otol Rhinol Laryngol. 2003;112(11):921-926.

14. Kao FC, Niu CC, Chen LH, Lai PL, Chen WJ. Maintenance of interbody space in one- and two-level anterior cervical interbody fusion: comparison of the effectiveness of autograft, allograft, and cage. Clin Orthop Relat Res. 2005(430):108-116. 
15. Jacobs W, Willems PC, Kruyt M, et al. Systematic review of anterior interbody fusion techniques for single- and double-level cervical degenerative disc disease. Spine (Phila Pa 1976). 2011;36(14):E950960.

16. Anderson KK, Arnold PM. Oropharyngeal Dysphagia after anterior cervical spine surgery: a review. Global Spine J. 2013;3(4):273-286.

17. Lee CK, Shin DA, Yi S, et al. Correlation between cervical spine sagittal alignment and clinical outcome after cervical laminoplasty for ossification of the posterior longitudinal ligament. $J$ Neurosurg Spine. 2016;24(1):100-107.

18. Uchida K, Nakajima H, Sato R, et al. Cervical spondylotic myelopathy associated with kyphosis or sagittal sigmoid alignment: outcome after anterior or posterior decompression. J Neurosurg Spine. 2009;11(5):521-528.

19. Fehlings MG, Gray R. Importance of sagittal balance in determining the outcome of anterior versus posterior surgery for cervical spondylotic myelopathy. J Neurosurg Spine. 2009;11(5):518-519; discussion 519-520.

20. Inoue T, Ando K, Kobayashi K, et al. Age-Related Changes in T1 and C7 Slope and the Correlation Between Them in More Than 300 Asymptomatic Subjects. Spine (Phila Pa 1976). 2020.

21. Lau D, DiGiorgio AM, Chan AK, et al. Applicability of cervical sagittal vertical axis, cervical lordosis, and T1 slope on pain and disability outcomes after anterior cervical discectomy and fusion in patients without deformity. J Neurosurg Spine. 2019:1-8.

22. Staub BN, Lafage R, Kim HJ, et al. Cervical mismatch: the normative value of T1 slope minus cervical Iordosis and its ability to predict ideal cervical lordosis. J Neurosurg Spine. 2018;30(1):31-37.

23. Miyata $M$, Neo M, Fujibayashi $S$, Ito $H$, Takemoto $M$, Nakamura T. O-C2 angle as a predictor of dyspnea and/or dysphagia after occipitocervical fusion. Spine (Phila Pa 1976). 2009;34(2):184-188.

24. Khaki F, Zusman NL, Nemecek AN, Ching AC, Hart RA, Yoo JU. Postoperative prevertebral soft tissue swelling does not affect the development of chronic dysphagia following anterior cervical spine surgery. Spine (Phila Pa 1976). 2013;38(9):E528-532.

25. Rinn JA, Kane J, Albert TJ, Vaccaro AR, Hilibrand AS. What is the incidence and severity of dysphagia after anterior cervical surgery? Clin Orthop Relat Res. 2011;469(3):658-665. 